\title{
Increased fruit and vegetable consumption improves antibody response to vaccination in older people: the ADIT study
}

\author{
J. D. Edgar ${ }^{1}$, A. Gibson ${ }^{2}$, C. E. Neville ${ }^{2}$, S. E. C. M. Gilchrist ${ }^{2}$, M. C. Mckinley $^{2}$, C. C. Patterson ${ }^{2}$, \\ I. S. Young ${ }^{2}$ and J. V. Woodside ${ }^{2}$ \\ ${ }^{1}$ Regional Immunology Service, Belfast Health and Social Care Trust, Belfast BT12 6BN, UK and ${ }^{2}$ School of Medicine, \\ Dentistry and Biomedical Sciences, Queen's University Belfast BT12 6BJ, UK
}

\begin{abstract}
The immune system undergoes a range of changes as individuals become older. Multiple underlying factors contribute to this immunological ageing, and in this study we have examined the possibility that inadequate diet may be a contributing factor. Fruit and vegetable intake, which can be low in the elderly, is associated with reduced chronic disease risk. This study tested the hypothesis that increased fruit and vegetable intake may positively affect clinically relevant measures of immune function.

Eighty-three healthy volunteers aged 65-85 years following a low fruit and vegetable diet ( $\leq 2$ portions/d) were recruited and randomised to continue following their normal diet, or to consume at least 5 portions of fruit and vegetables daily for 16 weeks. At 12 weeks, Tetanus toxoid ( $0.5 \mathrm{ml} \mathrm{IM})$ and Pneumovax II $(0.5 \mathrm{ml} \mathrm{IM})$ were administered. Specific antibody binding to tetanus toxoid (total IgG) and pneumococcal capsular polysaccharide (total IgG and IgG2) were assessed by ELISA at baseline, 12 and 16 weeks. Natural killer cell (NKC) cytotoxicity was assessed by flow cytometry at baseline, 12 and 16 weeks. Biochemical markers of nutritional status were assessed at baseline, 6, 12 and 16 weeks to monitor compliance.

Eighty-two participants completed the 16-week intervention. There was a significant difference in change in self-reported fruit and vegetable consumption between 2 and 5 portions/d groups (mean change in the number of portions (SD); 2 portions/d, 0.41 (0.79); 5 portions/d, $4.61(1.38) ; P<0.001)$, and this was reflected in significant increases in vitamin $C$, lutein, zeaxanthin, $\beta$-cryptoxanthin and lycopene status (data not shown). Antibody binding to pneumococcal capsular polysaccharide (total IgG and IgG2) increased more in the 5 portions/d group than in the 2 portions/d group (geometric mean $(95 \% \mathrm{CI}$ ) change from baseline which represents percentage of baseline; total IgG: 2 portions/d, 179 (142,227); 5 portions/d, $299(213,421) ; P=0.02$; IgG2: 2 portions/d, 186 (151,231); 5 portions/d, 304 (220,419); $P=0.01)$. There was no significant difference in antibody binding to tetanus toxoid between the two intervention groups. NKC cytotoxicity tended to be greater in the 5 portions/d group than in the 2 portions/d group at 16 weeks $(P=0.07)$, and this was similar when NKC cytotoxicity was adjusted for the proportion of NK cells $(P=0.07)$, but did not reach statistical significance.

In this study, there is a greater specific antibody response to Pneumovax II vaccination in those consuming 5 portions of fruit and vegetables per day compared with those consuming 2 portions of fruit and vegetables per day. Therefore increased fruit and vegetable intake may improve antibody response to vaccination in older people, linking an achievable dietary goal with a potential improvement in immune function.
\end{abstract}

